\title{
Contralateral adrenal thinning as a distinctive feature of mild autonomous cortisol excess of the adrenal tumors
}

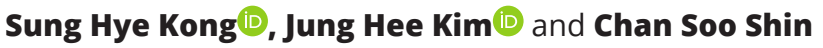 \\ Department of Internal Medicine, Seoul National University Hospital, Seoul National University College of Medicine, \\ Seoul, South Korea
}

Correspondence should be addressed to $\mathrm{H} \mathrm{Kim}$ Email jhkxingfu@gmail.com

\begin{abstract}
Objective: To identify radiologic features that correlate with mild autonomous cortisol excess using planar and volumetric analysis.

Design: Cross-sectional study.

Methods: In the study, 64 patients with overt Cushing syndrome (CS), 59 patients with mild autonomous cortisol excess (MACE), and 64 patients with nonfunctioning adrenal tumors (NFAT) with evaluable CT scans were included. Patients with NFAT and MACE were BMI-matched with those with overt CS. Planar and volumetric analyses of CT scans were performed in DICOM images using OsiriX software.

Results: The mean age was $56.6 \pm 1.01$ years, and 123 patients (65.1\%) were female. In the order of NFAT, MACE, and overt CS, the diameters and volumes of the adenoma increased, while limb widths and volumes of the contralateral adrenal gland decreased. Patients with MACE or overt CS were more likely to have osteoporosis than those with NFAT $(P=0.006)$, and patients with overt CS were more likely to experience a fragility fracture than those with NFAT or MACE $(P=0.002)$. Among radiologic features, the limb width of the contralateral adrenal gland correlated with the cortisol level after overnight dexamethasone suppression test $(r=-0.583, P<0.001)$. Conclusions: The study showed that the contralateral adrenal limb thinning was a distinctive radiologic feature of autonomous cortisol excess in the planar and volumetric analysis.
\end{abstract}

\section{Introduction}

Incidentally discovered adrenal adenomas have become a common finding in clinical practice, reaching almost $10 \%$ in the elderly $(1,2,3,4)$. Although approximately $80 \%$ of adrenal incidentalomas (AIs) are nonfunctioning adrenal tumors (NFAT), autonomous cortisol-secreting tumors account for approximately $12-30 \%$ of AIs (4, 5, 6). Autonomous cortisol-secreting tumors can manifest as overt Cushing syndrome (CS) or mild autonomous cortisol excess (MACE), defined as altered cortisol suppression after (c) 2020 European Society of Endocrinology Printed in Great Britain dexamethasone without overt CS manifestation $(4,7)$. In the past, adrenal tumors with MACE have been known to rarely develop overt CS $(8,9)$. However, recent evidence suggests that MACE might also be associated with an increased risk for diabetes mellitus or cardiovascular disease $(6,10,11)$. The European guideline suggested that surgical management can be considered if cortisol-related comorbidities, such as diabetes, hypertension, fracture, and obesity, are present (4). 
Tumors with overt CS distinctly show sizes mostly larger than $2 \mathrm{~cm}$, along with atrophy of the remaining contralateral adrenal gland due to suppressed adrenocorticotropic hormone (ACTH) production (12). Reports have shown that contralateral adrenal thinning helps distinguish tumors with overt CS from other adrenal diseases (13). It is still unclear whether the degree of contralateral adrenal thinning correlates with mild cortisol excess.

Most of the previous studies only analyzed the width of the adrenal gland (13). However, there have been reports that volumetric analysis is needed due to their non-elliptical shapes (14). A previous study based on volumetric analysis assumed the adrenal tumor to be an elliptical structure, and the result may miscalculate the real volume of adrenal tumors (15). Although there was a study with only a small number of patients on correlations between adrenal volume and clinical features (16), more studies are needed to validate these findings.

In this regard, we compared CT imaging characteristics among patients according to the extent of cortisol secretion using volumetric and planar analyses and determined that anatomical changes were found in patients with MACE.

\section{Methods}

\section{Study design and participants}

From January 2004 to November 2019 at Seoul National University Hospital, 3843 patients were diagnosed with adrenal incidentaloma. After the exclusion of patients with pheochromocytoma $(n=219)$, primary aldosteronism $(n=360)$, and other factors such as adrenocortical carcinoma or metastasis $(n=187), 2692$ patients with NFAT and 385 patients with an autonomous cortisol-secreting adrenal mass remained. Among 385 patients with an autonomous cortisol-secreting adrenal mass, 96 patients had overt CS. Of 96 patients with overt CS, 32 patients were excluded since they did not have abdomen-pelvis, kidney, or adrenal CT scans compatible for analysis using the OsiriX software. For the remaining 64 patients, the same number of BMI-matched patients with NFAT and MACE were included for comparison. Among them, five patients were excluded from the final analysis due to a lack of ACTH level data. The final analysis consisted of 187 patients, 64 patients with overt CS, 59 patients with MACE, and 64 patients with NFAT (Fig. 1). Plasma ACTH levels were reviewed in the selected patients to exclude the possibility of Cushing's disease, and all included patients had ACTH levels lower than

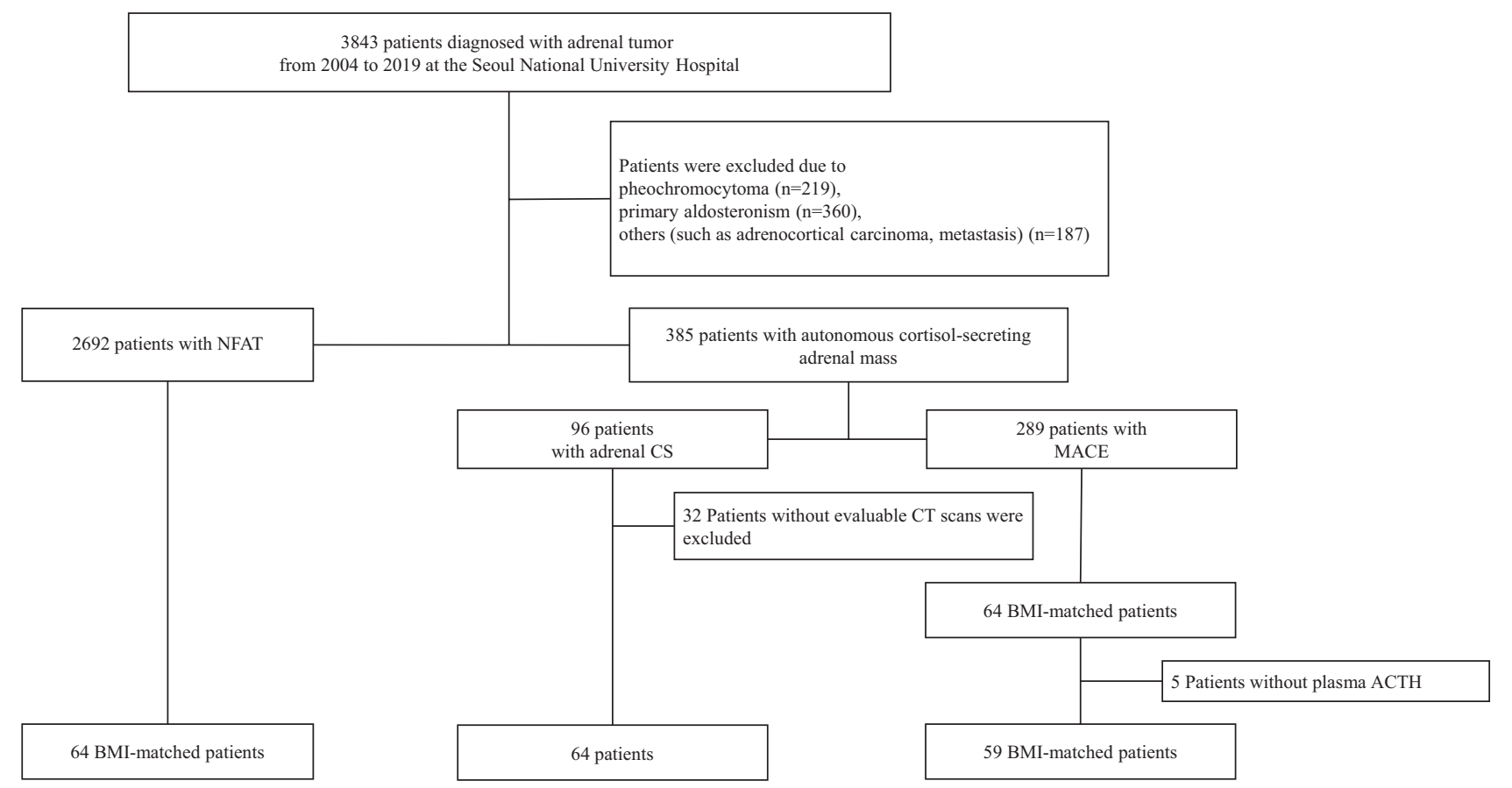

Figure 1

Patient selection. NFAT, non-functioning adrenal tumor; CS, Cushing syndrome; MACE, mild autonomous cortisol excess. 
the upper normal level $(60.0 \mathrm{pg} / \mathrm{mL})$. Anthropometric measures, past medical history, and laboratory data were retrospectively reviewed using electronic medical records. Diabetes mellitus was defined according to the criteria suggested by the American Diabetes Association: fasting plasma glucose concentration $\geq 7.0 \mathrm{mmol} / \mathrm{L}(126 \mathrm{mg} / \mathrm{dL})$, 2-h plasma glucose $\geq 11.1 \mathrm{mmol} / \mathrm{L}(200 \mathrm{mg} / \mathrm{dL})$, HbA1c $\geq 6.5 \%$, or currently on oral antidiabetic drug or insulin (17). Hypertension was defined as systolic and diastolic blood pressure of $\geq 140 / 90 \mathrm{mmHg}$ or currently on antihypertensive medication. All patients with MACE and overt CS and 56 patients with NFAT had their blood pressure measured more than once. Among patients with NFAT, eight patients had their blood pressure measured once and were not on anti-hypertensive medication, and those patients were considered non-hypertensive. Osteoporosis was defined as having a $T$-score below -2.5 in postmenopausal women and men aged $\geq 50$ years and $Z$-scores below -2.0 in younger women and men from dual-energy X-ray absorptiometry (18). Bone densitometry scans were performed in 35,47 , and 48 patients with NFAT, MACE, and overt CS, respectively. Fragility fracture event was defined as a fracture caused by minimal trauma, such as a fall from standing height or less. It includes patients' reports and morphometric fractures from X-rays. Spine $\mathrm{X}$-rays were performed in 39,49 , and 50 patients with NFAT, MACE, and overt CS, respectively. Ever-smokers were defined as those patients who had records of smoking history of $>5$ packs of cigarettes during their lifetime. The study was approved by the institutional review board of the Seoul National University Hospital (IRB No. 1801-010911), conducted according to the Declaration of Helsinki.
Informed consent was waived because of the retrospective study design.

\section{CT examinations}

A total of 187 patients underwent abdomen-pelvis $(n=109)$, kidney $(n=32)$, or adrenal $(n=46)$ CT protocols with pre-contrast images using the dual-energy scanning mode of a dual-source CT scanner (Canon ONE Aquilion for abdomen-pelvis CT, GE LightSpeed Ultra 8 for kidney CT, Philips IQon Spectral CT for adrenal CT). Pre-contrast images were obtained in each protocol to evaluate the radiologic features of the adrenal glands. Axial images were reconstructed at slice thicknesses of $3,2.5$, and 3 $\mathrm{mm}$ with a reconstruction interval of $3,1.4$, and $3 \mathrm{~mm}$ in the abdomen-pelvis, kidney, and adrenal CT protocols, respectively. Tube voltages and maximum tube currents were 100, 100, and $120 \mathrm{kV}$ and 510, 355, and $192 \mathrm{~mA}$ for abdomen-pelvis, kidney, and adrenal CT protocols, respectively.

\section{Measurements of radiologic features}

For volumetric measurements of the adrenal glands, we used OsiriX, an imaging software dedicated to analyzing DICOM images (Pixmeo SARL, Bernex, Switzerland, www. osirixviewer.com). De-identified images were transferred in DICOM format to the OsiriX database. Volumetric analysis was performed using axial slices, outlined manually on each slice at 6- to 12.5-fold magnification. Volumes were then determined separately for the left and right adrenal glands by software output in OsiriX (Fig. 2A). Widths of
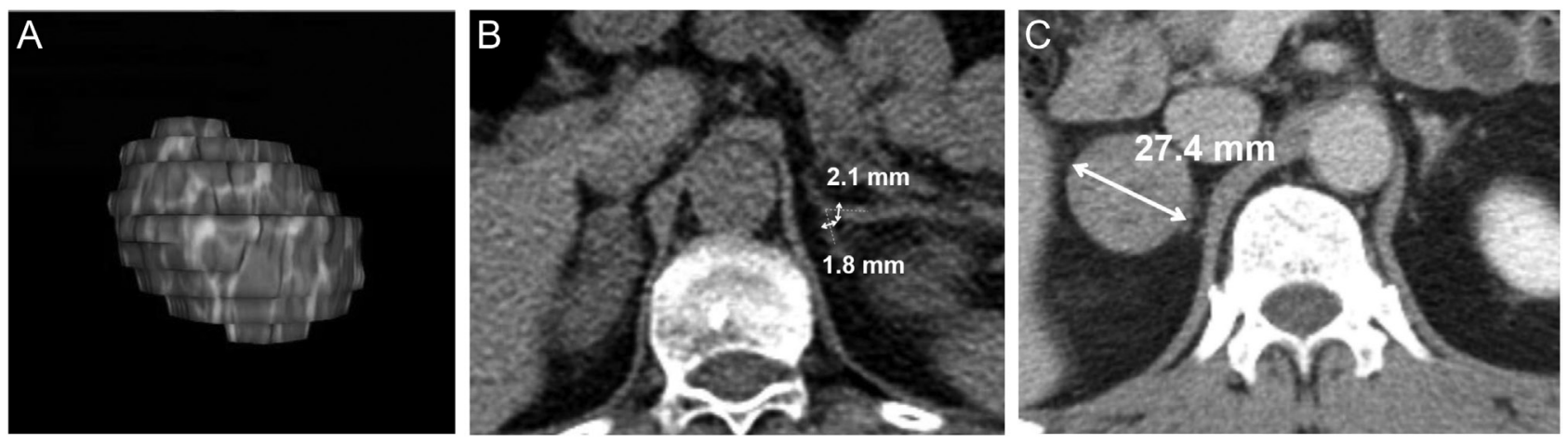

\section{Figure 2}

Measurement of width and volume of adrenal glands. (A) Volumetric analysis of the adrenal gland, (B) width of the contralateral adrenal limbs, and $(C)$ the mass. $(A)$ Volumetric modeling was generated using OsiriX software with DICOM images. It was performed using axial slices outlined manually on each slice. (B) Widths of the contralateral adrenal limbs were measure as maximum widths of the limbs perpendicular to the long axis. (C) The width of the mass was measured as the maximum width. 
the medial and lateral adrenal limbs were measured as the maximum width of the limbs perpendicular to the long axis, as previously suggested by Vincent et al. (19) (Fig. 2B). Diameters of the mass were measured as the maximum width of the gland (Fig. 2C). The widths of the contralateral adrenal limb were defined as the mean of the medial and lateral limb widths. Attenuation values were measured in Hounsfield Units (HU) from precontrast images in the middle of the adrenal glands. The CT scans were reviewed by two different investigators ( $\mathrm{S} \mathrm{H} \mathrm{K}$ and $\mathrm{J} \mathrm{H} \mathrm{K}$ ) who were not given information about the patients or the patients' hormonal results. If there was a disagreement on measurements $(>0.1 \mathrm{~cm}$ of diameter, $>0.1 \mathrm{~cm}^{3}$ of volume), investigators re-reviewed the CT scans together and measured them again. All measurements were performed at an institutional standard window setting for abdominal CT scans (width: $360 \mathrm{HU}$, level: $60 \mathrm{HU})$.

\section{Definition of NFAT, MACE, and overt CS}

NFAT was defined as a condition with a normally suppressed cortisol level of $\leq 1.8 \mu \mathrm{g} / \mathrm{dL}$ after ODST without primary aldosteronism and pheochromocytoma. MACE was defined as a serum cortisol level $>1.8 \mu \mathrm{g} / \mathrm{dL}$ after the $1 \mathrm{mg}$ ODST with 24-h urine-free cortisol levels within the reference range and without typical Cushingoid features such as easy bruising, moon face, buffalo hump, or central obesity (4). Overt CS, known as overt Cushing syndrome, was defined as a condition with typical Cushingoid features without suppression of serum cortisol after ODST as well as 24-h urine-free cortisol levels greater than the upper limit of the normal range (ULN).

\section{Laboratory data}

Serum cortisol and 24-h urine-free cortisol levels were measured using a RIA kit (Cisbio Bioassays, France, from January 1999 to April 2017; and IMMUNOTEC, Czech Republic, since April 2017) with intra- and interassay coefficients of variation (CV) of $5.8 \%$ and $9.2 \%$, respectively. The reference ranges for the 24-h urine-free cortisol were 13.8-75.4 $\mu \mathrm{g} /$ day after April 2017 and 19.4$115.2 \mu \mathrm{g} /$ day from January 1999 to March 2017. Plasma ACTH was measured by an IRMA (CIS-Bio International, Saclay, France) with a reference range of 10.0-60.0 pmol/L. The intra- and inter-assay CVs of ACTH used were 3.7\% and $3.8 \%$, respectively (9).

\section{Statistical analyses}

Continuous variables are expressed as mean \pm s.D. and categorical variables as numbers (percentages). Comparisons between groups were analyzed by performing Student's $t$-test in a normal distribution, Kruskal-Wallis test in a non-normal distribution for continuous variables, and $\chi^{2}$ test for categorical variables. For each variable, patients with missing data values were excluded from the analyses. Patients were matched for BMI among the NFAT, MACE, and CPA groups using a propensity score matching method with the MatchIt package from R. Correlation between contralateral limb width and cortisol levels was analyzed using linear regression. Area under the curve (AUC) of the diameters and volumes of each adrenal gland was calculated through pairwise comparison of the receiver operator curve with Delong's test. All analyses were performed using the $\mathrm{R}$ language and environment for statistical computing. Statistical significance was indicated as $P<0.05$ for all tests.

\section{Results}

\section{Clinical characteristics of the patients}

Of the 187 patients, mean age was $56.6 \pm 1.0$ years, and the majority were female (65.1\%). As described in Table 1 , patients with overt CS were younger than those with NFAT or MACE, wherein ages between patients with NFAT and MACE are comparable. The location of adrenal mass was similar among groups. Attenuation values in precontrast CT imaging were higher in patients with overt CS than in those with NFAT or MACE. Patients with overt CS had the highest cortisol after ODST and 24-h urinefree cortisol levels among groups (post-ODST cortisol of $5.6 \pm 3.9,1.0 \pm 0.6$, and $16.0 \pm 8.3 \mu \mathrm{g} / \mathrm{dL}$; and 24 -h urinefree cortisol of $0.55 \pm 0.26,0.64 \pm 0.17,4.63 \pm 4.88 \times \mathrm{ULN}$, for MACE, NFAT, and Overt CS, respectively). Patients with overt CS were more likely to have osteoporosis than those with NFAT $(9$ (14.1\%) and 1 (1.6\%) for overt CS and NFAT, respectively; $P=0.006)$ and more likely to have hypertension (33 (51.6\%), 16 (45.8\%), and 19 (29.7\%) for overt CS, MACE, and NFAT, respectively; $P=0.019)$ and fragility fracture events than those with MACE or NFAT (6 (9.4\%), $0(0 \%)$, and $0(0 \%)$ for overt CS, MACE, and NFAT, respectively; $P=0.002$ ). Among the six patients with fragility fractures, all patients underwent spine X-rays, and three of them were asymptomatic. The proportion of ever-smokers and other comorbidities, such as angina, diabetes, dyslipidaemia, or stroke, had a similar likelihood among the groups. 
Table 1 Clinical and radiologic characteristics of study participants.

\begin{tabular}{l}
\hline Variables \\
\hline Cortisol after ODST ( $\mu \mathrm{g} / \mathrm{dL})$ \\
Age (years) \\
Female \\
BMI $\left(\mathrm{kg} / \mathrm{m}^{2}\right)$ \\
Ever-smoker \\
ACTH (pg/mL) \\
24-h urine cortisol (xULN) \\
24-h urine cortisol ( $\mu \mathrm{g} / \mathrm{day})$ \\
Angina \\
DM \\
HTN \\
Dyslipidemia \\
Osteoporosis \\
Fragility fracture \\
Stroke \\
Location of adrenal mass (left) \\
CT attenuation value (HU) \\
Mass diameter (mm) \\
Mass volume (cm ${ }^{3}$ ) \\
Contralateral diameter (mm) \\
Contralateral volume (cm ${ }^{3}$ ) \\
\hline
\end{tabular}

\begin{tabular}{c} 
NFAT $(n=64)$ \\
\hline $1.0 \pm 0.6$ \\
$57.6 \pm 12.4$ \\
$39(60.9 \%)$ \\
$25.6 \pm 4.1$ \\
$0 / 13(0 \%)$ \\
$21.7 \pm 10.6$ \\
$0.64 \pm 0.17$ \\
$72.1 \pm 20.7(n=14)$ \\
$2(3.1 \%)$ \\
$15(23.4 \%)$ \\
$19(29.7 \%)$ \\
$16 / 50(32.0 \%)$ \\
$1 / 35(2.9 \%)$ \\
$0 / 39(0.0 \%)$ \\
$1(1.6 \%)$ \\
$41(65.1 \%)$ \\
$11.8 \pm 23.4$ \\
$20.9 \pm 9.4$ \\
$5.4 \pm 3.1$ \\
$3.5 \pm 1.2$ \\
$1.4 \pm 0.8$
\end{tabular}

\begin{tabular}{c}
\hline MACE $(n=59)$ \\
\hline $5.6 \pm 3.9$ \\
$61.6 \pm 13.0$ \\
$36(61.0 \%)$ \\
$25.3 \pm 3.8$ \\
$1 / 14(7.1 \%)$ \\
$12.6 \pm 11.9$ \\
$0.55 \pm 0.26$ \\
$56.0 \pm 29.3(n=42)$ \\
$3(5.1 \%)$ \\
$16(27.1 \%)$ \\
$27(45.8 \%)$ \\
$15 / 58(25.8 \%)$ \\
$11 / 47(23.4 \%)$ \\
$0 / 49(0.0 \%)$ \\
$0(0.0 \%)$ \\
$32(54.2 \%)$ \\
$16.1 \pm 17.7$ \\
$26.0 \pm 5.6$ \\
$5.9 \pm 5.8$ \\
$2.6 \pm 1.0$ \\
$1.2 \pm 0.8$
\end{tabular}

\begin{tabular}{c}
\hline Overt CS $(n=64)$ \\
\hline $16.0 \pm 8.3$ \\
$50.5 \pm 13.5$ \\
$48(75.0 \%)$ \\
$24.7 \pm 3.7$ \\
$1 / 19(5.2 \%)$ \\
$15.9 \pm 11.4$ \\
$4.63 \pm 4.88$ \\
$534.3 \pm 564.7(n=64)$ \\
$4(6.2 \%)$ \\
$15(23.4 \%)$ \\
$33(51.6 \%)$ \\
$15 / 59(25.4 \%)$ \\
$9 / 48(18.8 \%)$ \\
$6 / 50(12.0 \%)$ \\
$4(6.2 \%)$ \\
$41(65.1 \%)$ \\
$23.4 \pm 13.1$ \\
$30.4 \pm 8.2$ \\
$11.4 \pm 13.1$ \\
$2.2 \pm 0.9$ \\
$1.0 \pm 0.8$
\end{tabular}

\begin{tabular}{c}
\hline P-value \\
\hline- \\
$0.003^{\mathrm{bc}}$ \\
0.130 \\
0.280 \\
0.792 \\
0.180 \\
$<0.001^{\mathrm{bc}}$ \\
$<0.001^{\mathrm{bc}}$ \\
0.676 \\
1.000 \\
$0.019^{\mathrm{ac}}$ \\
0.850 \\
$0.010^{\mathrm{ac}}$ \\
$0.001^{\mathrm{bc}}$ \\
$0.076^{\mathrm{bc}}$ \\
1.000 \\
$0.001^{\mathrm{bc}}$ \\
$<0.001^{\mathrm{abc}}$ \\
$<0.001^{\mathrm{abc}}$ \\
$<0.001^{\mathrm{bcc}}$ \\
$0.003^{\mathrm{c}}$
\end{tabular}

CS, Cushing syndrome; DM, diabetes mellitus; HTN, hypertension; MACE, mild autonomous cortisol excess; NFAT, nonfunctioning adrenal tumor; ODST, overnight dexamethasone suppression test; ULN, upper limit of normal range.

Continuous variables are expressed as mean \pm S.D. and categorical variables as numbers (percentages). Comparisons between groups were analyzed by performing a Student $t$-test, whereas a $\chi^{2}$ test was used for categorical variables. ${ }^{a} P<0.05$ between NFAT and MACE; ${ }^{b}$ MACE and CS; ${ }^{c}$ NFAT and CS according to post hoc Bonferroni analysis. Normal range of ACTH was 10.0-60.0 pg/mL, and normal range of 24-h urine cortisol was $19.5-115.6 \mu \mathrm{g} / \mathrm{day}$ from January 1999 to March 2017 and 13.8-75.4 $\mu$ g/day since April 2017.

\section{Radiologic features of the adrenal glands}

Patients with MACE had shorter diameters of the mass than those with overt CS and longer diameters than those with NFAT $(26.0 \pm 5.6,30.3 \pm 8.2,21.2 \pm 10.2 \mathrm{~mm}$, respectively; $P<0.001$, Table 1). Inversely, patients with MACE had wider contralateral adrenal gland limb width than those with overt CS, but had thinner limb width than those with NFAT $(2.6 \pm 1.0,1.9 \pm 0.8,3.4 \pm 0.9 \mathrm{~mm}$, respectively; $P<0.001)$.

Table 2 Diagnostic accuracy of radiologic features for differentiation of autonomous cortisol excess of the adrenal glands between patients with NFAT and MACE.

\begin{tabular}{l}
\hline Radiological features \\
\hline Diameter $(\mathrm{mm})$ \\
Mass \\
Contralateral \\
Volume (cm3) \\
Mass \\
Contralateral \\
\hline
\end{tabular}

\begin{tabular}{l}
\hline AUC \\
\hline 0.73 \\
0.83 \\
\\
0.65 \\
0.62 \\
\hline
\end{tabular}

\begin{tabular}{c}
\hline $\mathbf{9 5 \%} \mathbf{~ C l}$ \\
\hline $0.64-0.81$ \\
$0.76-0.88$ \\
\\
$0.57-0.74$ \\
$0.54-0.71$ \\
\hline
\end{tabular}

\begin{tabular}{c} 
P-value \\
\hline $0.001^{*}$ \\
$<0.001$ \\
$0.001^{*}$ \\
$0.006^{*}$ \\
\hline
\end{tabular}

AUC, area uner the curve; $\mathrm{Cl}$, confidence interval; MACE, mild autonomous cortisol excess; NFAT, nonfunctioning adrenal tumor. Autonomous cortisol excess was defined as cortisol level $>1.8 \mu \mathrm{g} / \mathrm{dL}$ after overnight dexamethasone suppression test. $* P<0.05$ in Delong's test compared with the limb width of the contralateral adrenal gland.
In volumetric analysis, volumes of the mass were smaller in patients with MACE than in those with overt CS, but larger than in those with NFAT $(5.86 \pm 5.81$, $11.41 \pm 13.10,5.37 \pm 3.13 \mathrm{~cm}^{3}$, respectively; $P=0.001$ between overt CS and MACE, $P<0.001$ between MACE and NFAT). Contralateral adrenal gland volumes were larger in patients with NFAT than in those with overt CS $\left(1.41 \pm 0.87,1.17 \pm 0.76 \mathrm{~cm}^{3}\right.$, respectively; $\left.P=0.009\right)$.

To find the best radiologic feature to detect autonomous cortisol excess of the adrenal glands, we evaluated the performance of each radiologic feature in AUCs, as shown in Table 2. Among radiologic features, the diameter of the contralateral adrenal limb had better AUC of 0.83 and $95 \%$ CI of $0.76-0.88(P<0.001)$ than the measured contralateral limb volume, mass diameter, and mass volume (Delong's test compared with the diameter of the contralateral adrenal limb, $P=0.045, P=0.001$, and $P<0.001$, respectively).

\section{Correlation between limb width of the contralateral gland and cortisol level}

There was a negative linear correlation between limb width of the contralateral adrenal gland and cortisol level 
after ODST $(r=-0.583, P<0.001)$, which indicates that the thinner the limb width of the contralateral adrenal gland, the higher the autonomous cortisol excess (Fig. 3). The corresponding cortisol after ODST to contralateral limb diameter of $2.5 \mathrm{~mm}$ was $3.5 \mu \mathrm{g} / \mathrm{dL}$. However, the diameters of the mass and cortisol levels after ODST had a positive correlation $(r=0.289, P=0.003)$, while the volumes of the mass and contralateral adrenal glands had an insignificant correlation with cortisol level after ODST.

\section{Discussion}

The study found that the diameter of the adrenal mass increased and the limb width of the contralateral side decreased in the order of NFAT, MACE, and overt CS. In volumetric analysis, volumes of adrenal mass were larger and volumes of the contralateral side were smaller in patients with MACE or overt CS than in those with NFAT. Among the radiologic features, the limb width of the contralateral adrenal gland was well correlated with the post-ODST cortisol level.

The key finding of the study is that the limb width of the contralateral adrenal gland was correlated with the cortisol level, even in patients with MACE. It has been reported that atrophy of the contralateral adrenal gland can be a stigma for overt hypercortisolism due to negative feedback (12). A recent study suggested that most patients with overt CS had a contralateral adrenal

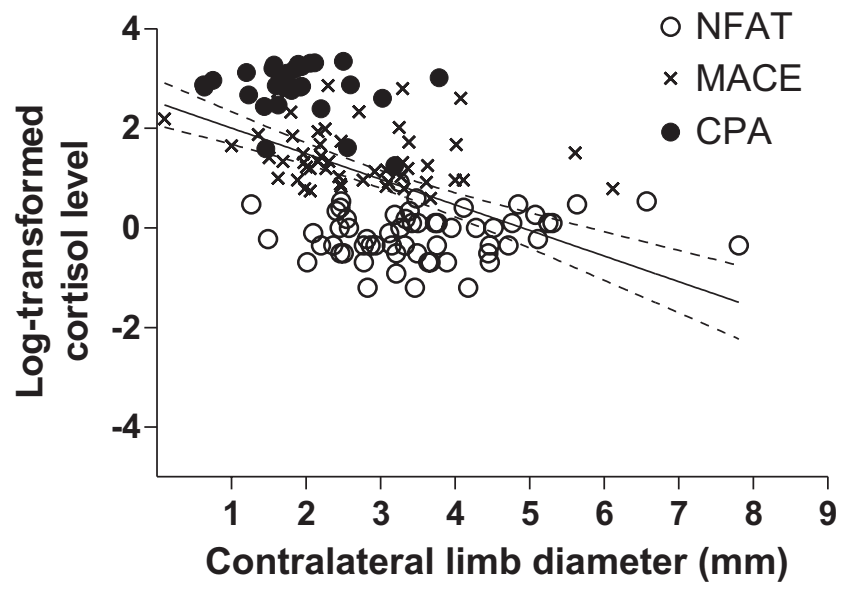

\section{Figure 3}

Correlation between contralateral limb diameter and cortisol level after ODST. Linear correlation analysis was done. ODST, overnight dexamethasone suppression test; NFAT, nonfunctioning adrenal tumor; MACE, mild autonomous cortisol excess; CS, Cushing syndrome. gland limb width thinner than $2 \mathrm{~mm}$, while patients with primary aldosteronism did not (13). However, due to the lack of patients with NFAT or MACE, the study could not show a serial correlation between the limb width and the degree of cortisol excess. Another study showed the clinical significance of the limb width of the contralateral adrenal glands in patients with MACE (15). In the study, the thinner the limb width, the longer the postoperative steroid replacement was in these patients (15). Among patients with MACE, the degree of cortisol excess was more severe in those with thinner limb widths, which correlates with the results of our study (15). It was also observed that the size of the mass was positively correlated with autonomous cortisol excess. This result was similar to previous studies, including ours, which showed positive correlations between the size of the mass and hypercortisolemia $(13,14)$.

The main pathophysiology of the size of the adrenal glands is hypothalamic-pituitary-adrenal axis suppression, resulting in an impaired stress response to metabolic illness (20). As metabolic diseases can be managed by active surveillance and intervention, early confirmation of MACE is a key to the management of these patients. However, the diagnosis of patients with MACE is still in debate. The ODST is a sensitive and well-designed traditional screening test to exclude MACE (21), but it has relatively poor specificity (70$80 \%)$, which requires additional diagnostic testing (22, 23). Measurement of 24-h urinary-free cortisol is also a sensitive test, but it could be falsely positive when patients are obese and falsely negative in patients with mild hypercortisolism or impaired renal function (24). Additionally, plasma ACTH levels are known to be suppressed in MACE, but overlap with healthy individuals has been reported (25), which could be due to the pulsatile secretion and short half-life of ACTH (26). Therefore, according to the recent European guidelines in 2016, the accompanying comorbidities, along with biochemical tests, can be considered when deciding whether to intervene in MACE patients (4). The size difference in the unaffected adrenal glands due to sustained suppression of central ACTH might be helpful in determining the management of MACE, along with comorbidities and biochemical tests.

Volumetric analysis based on 3D CT imaging was expected to provide better diagnostic insight than planar analysis in adrenal disease (27). This is because the shape of the adrenal gland is usually irregular and can hardly be assumed elliptical. However, regarding the contralateral adrenal gland, the limb width was better than the volume 
in differentiating early autonomous cortisol excess. This could be because the limb of the contralateral gland was too slender, like a thread, and irregular to measure volumetrically in patients with MACE or overt CS, while the width was relatively consistent to measure. Since the mass was usually elliptical, both diameters and volumes showed similarly acceptable results in distinguishing MACE from NFAT. It is clinically meaningful that the width from a basic image viewer system could be enough to estimate mild cortisol excess without volumetric analysis. Therefore, limb width measurement may aid in estimating the degree of cortisol excess.

Additionally, ACTH levels were similar among patients with NFAT, MACE, and CPA, possibly because of the low reproducibility and high inter-assay variability of plasma ACTH assays $(28,29)$. Additionally, ACTH has a diurnal variation, which implies that the timing of the measurement can affect the result (30). As our team has previously reported, plasma ACTH may have limited diagnostic value in diagnosing overt CS (25). In the report, plasma ACTH significantly overlapped between patients with overt CS and those with Cushing disease and only $33.3 \%$ of patients with adrenal Cushing syndrome had plasma ACTH $<2.2 \mathrm{pmol} / \mathrm{L}$ (25). Hence, as in our study, patients with overt CS could have higher plasma ACTH levels than expected. Although low ACTH production is one of the main reasons for adrenal atrophy in the contralateral adrenal gland, a single ACTH test may not be enough to reflect the patients' overall ACTH stimulation to the adrenal gland due to the diurnal variation of ACTH and the instability of the assay. However, further studies with a larger number of patients are needed to support this finding.

In terms of diabetes, $23.4 \%$ of patients with NFAT had diabetes, which was a higher rate than that of the general Korean population (19.6\% of men, $12.4 \%$ of women in their 50s) (31). Recently, Reimondo and colleagues reported that the prevalence of diabetes was higher in patients with adrenal incidentaloma than in those without (32). It has been suggested that adrenal adenomas may aggravate insulin resistance (33), and vice versa, insulin resistance may predispose patients to the occurrence of adrenal adenomas (34). On the other hand, the prevalence of diabetes was similar among groups. This might be because the prevalence of diabetes in patients with NFAT was higher than that in a previous report (32), while the prevalence in patients with MACE was similar to that in previous reports $(35,36)$. Since the analyzed population is based on patients who visited the tertiary hospital, participants in the study could be more likely to have comorbidities.

The study has several strengths. Thorough radiologic analysis with both planar and volumetry of both adrenal glands was performed. Performance of planar and volumetric analyses was also compared. The study included not only patients with overt CS but also those with MACE, which helped in correlating radiologic features with mild cortisol excess. BMI was matched among groups to reduce the effect of body size on adrenal size. However, there were some limitations to this study. The study was cross-sectional and could not evaluate the correlation between radiologic features and prognosis of the disease. The number of patients in the control group (NFAT) might be insufficient to see differences in clinical features. Additionally, although weight is the main factor associated with the size of normal adrenal glands, age and sex can influence the size as well (27, 37). Since the study was BMI-matched, not age- and sex-matched, those influences could affect the results. Nonetheless, patients with MACE were older than those with overt CS but similar in those with NFAT; thus, age may not interfere with distinguishing MACE from NFAT. The prevalence of hypertension could be underestimated due to the retrospective design of the study. Some patients with hypertension could be missed if their blood pressure was measured only once. Additionally, we assumed that patients who did not have their blood pressure measured did not have hypertension if they were not taking medication for hypertension, which could contribute to underestimation of the hypertension prevalence, especially in patients with NFAT. A total of three types of CT protocols were included in the study, which may affect volume or diameter measurements. However, the difference could be minimal because the thicknesses of each CT protocol were similar between 2.5 and $3 \mathrm{~mm}$, which included four to five images.

To conclude, the study suggested a negative correlation of the limb width of the contralateral adrenal gland with the degree of autonomous cortisol excess. Additionally, planar measurements from the CT scan were sufficiently sensitive without volumetric measurements. Further prospective studies are recommended to determine whether radiologic features of MACE can predict clinical outcomes of patients.

\section{Declaration of interest}

The authors declare that there is no conflict of interest that could be perceived as prejudicing the impartiality of the research reported. 


\section{Funding}

The research did not receive any specific grant from any funding agency in the public, commercial, or not-for-profit sector.

\section{References}

1 Young WF, Jr. Clinical practice. The incidentally discovered adrenal mass. New England Journal of Medicine 2007356 601-610. PubMed PMID: 17287480. (https://doi.org/10.1056/NEJMcp065470)

2 Davenport C, Liew A, Doherty B, Win HHN, Misran H, Hanna S, Kealy D, Al-Nooh F, Agha A, Thompson CJ, et al. The prevalence of adrenal incidentaloma in routine clinical practice. Endocrine 201140 80-83. (https://doi.org/10.1007/s12020-011-9445-6)

3 Kloos RT, Gross MD, Francis IR, Korobkin M \& Shapiro B. Incidentally discovered adrenal masses. Endocrine Reviews 199516 460-484. PubMed PMID: 8521790. Epub 1995/08/01. (https://doi. org/10.1210/edrv-16-4-460)

4 Fassnacht M, Arlt W, Bancos I, Dralle H, Newell-Price J, Sahdev A, Tabarin A, Terzolo M, Tsagarakis S \& Dekkers OM. Management of adrenal incidentalomas: European society of endocrinology clinical practice guideline in collaboration with the European network for the study of adrenal tumors. European Journal of Endocrinology 2016 175 G1-G34. (https://doi.org/10.1530/EJE-16-0467)

5 Mantero F, Terzolo M, Arnaldi G, Osella G, Masini AM, Alt A, Giovagnetti M, Opocher G \& Angeli A. A survey on adrenal incidentaloma in Italy. Study group on adrenal tumors of the Italian society of endocrinology. Journal of Clinical Endocrinology \& Metabolism 200085 637-644. (https://doi.org/10.1210/ jcem.85.2.6372)

6 Di Dalmazi G, Vicennati V, Garelli S, Casadio E, Rinaldi E, Giampalma E, Mosconi C, Golfieri R, Paccapelo A, Pagotto U, et al. Cardiovascular events and mortality in patients with adrenal incidentalomas that are either non-secreting or associated with intermediate phenotype or subclinical Cushing's syndrome: a 15-year retrospective study. Lancet Diabetes Endocrinology 20142 396-405. (https://doi.org/10.1016/S2213-8587(13)70211-0)

7 Lee JM, Kim MK, Ko SH, Koh JM, Kim BY, Kim SW, Kim SK, Kim HJ, $\mathrm{Ryu} \mathrm{OH}$, Park J, et al. Clinical guidelines for the management of adrenal incidentaloma. Endocrinology \& Metabolism 201732 200-218. (https://doi.org/10.3803/EnM.2017.32.2.200)

8 Terzolo M, Bovio S, Reimondo G, Pia A, Osella G, Borretta G \& Angeli A. Subclinical Cushing's syndrome in adrenal incidentalomas. Endocrinology \& Metabolism Clinics of North America 200534 423-439. PubMed PMID: 15850851. Epub 2005/04/27. (https://doi. org/10.1016/j.ecl.2005.01.008)

9 Hong AR, Kim JH, Park KS, Kim KY, Lee JH, Kong SH, Lee SY, Shin CS, Kim SW \& Kim SY. Optimal follow-up strategies for adrenal incidentalomas: reappraisal of the 2016 ESE-ENSAT guidelines in real clinical practice. European Journal of Endocrinology 2017177 475-483. PubMed PMID: 28870984. Epub 2017/09/06. (https://doi. org/10.1530/EJE-17-0372)

10 Debono M, Bradburn M, Bull M, Harrison B, Ross RJ \& NewellPrice J. Cortisol as a marker for increased mortality in patients with incidental adrenocortical adenomas. Journal of Clinical Endocrinology \& Metabolism 201499 4462-4470. PubMed PMID: 25238207. Pubmed Central PMCID: PMC4255126. Epub 2014/09/23. (https:// doi.org/10.1210/jc.2014-3007)

11 Morelli V, Reimondo G, Giordano R, Della Casa S, Policola C, Palmieri S, Salcuni AS, Dolci A, Mendola M, Arosio M, et al. Longterm follow-up in adrenal incidentalomas: an Italian multicenter study. Journal of Clinical Endocrinology \& Metabolism 201499 827-834. PubMed PMID: 24423350. Epub 2014/01/16. (https://doi. org/10.1210/jc.2013-3527)
12 Kyle LH, Meyer RJ \& Canary JJ. Mechanism of adrenal atrophy in Cushing's syndrome due to adrenal tumor. New England Journal of Medicine 1957257 57-61. (https://doi.org/10.1056/ NEJM195707112570203)

13 Park SY, Oh YT, Jung DC \& Rhee Y. Prediction of adrenal adenomas with hypercortisolism by using adrenal computed tomography: emphasis on contralateral adrenal thinning. Journal of Computer Assisted Tomography 201539 741-746. (https://doi.org/10.1097/ RCT.0000000000000269)

14 Serifoglu I, Oz II \& Bilici M. The adrenal gland volume measurements in manifestation of the metabolic status in type-2 diabetes mellitus patients. International Journal of Endocrinology 201620167195849. (https://doi.org/10.1155/2016/7195849)

15 Sugiura M, Imamura Y, Kawamura K, Yamamoto S, Sazuka T, Nakamura K, Sakamoto S, Nagano H, Koide H, Tanaka T, et al. Contralateral adrenal width predicts the duration of prolonged post-surgical steroid replacement for subclinical Cushing syndrome. International Journal of Urology 201825 583-588. (https://doi. org/10.1111/iju.13566)

16 Amsterdam JD, Marinelli DL, Arger P \& Winokur A. Assessment of adrenal gland volume by computed tomography in depressed patients and healthy volunteers: a pilot study. Psychiatry Research 198721 189-197. (https://doi.org/10.1016/0165-1781(87)90022-9)

17 American Diabetes Association. Classification and diagnosis of diabetes: standards of medical care in Diabetes-2020. Diabetes Care 202043 (Supplement 1) S14-S31. (https://doi.org/10.2337/ dc20-S002)

18 World Health Organization. Assessment of Fracture Risk and Its Application to Screening for Postmenopausal Osteoporosis: Report of a WHO Study Group [Meeting Held in Rome from 22 to 25 June 1992]. World Health Organization, 1994.

19 Vincent JM, Morrison ID, Armstrong P \& Reznek RH. The size of normal adrenal glands on computed tomography. Clinical Radiology 199449 453-455. (https://doi.org/10.1016/s0009-9260(05)81739-8)

20 Di Dalmazi G, Vicennati V, Rinaldi E, Morselli-Labate AM, Giampalma E, Mosconi C, Pagotto U \& Pasquali R. Progressively increased patterns of subclinical cortisol hypersecretion in adrenal incidentalomas differently predict major metabolic and cardiovascular outcomes: a large cross-sectional study. European Journal of Endocrinology 2012166 669-677. (https://doi.org/10.1530/ EJE-11-1039)

21 Terzolo M, Stigliano A, Chiodini I, Loli P, Furlani L, Arnaldi G, Reimondo G, Pia A, Toscano V, Zini M, et al. AME position statement on adrenal incidentaloma. European Journal of Endocrinology 2011164 851-870. (https://doi.org/10.1530/EJE-101147)

22 Nieman LK, Biller BM, Findling JW, Newell-Price J, Savage MO, Stewart PM \& Montori VM. The diagnosis of Cushing's syndrome: an endocrine society clinical practice guideline. Journal of Clinical Endocrinology \& Metabolism 200893 1526-1540. (https://doi. org/10.1210/jc.2008-0125)

23 Reimondo G, Allasino B, Bovio S, Saba L, Ardito A, Angeli A \& Terzolo M. Pros and cons of dexamethasone suppression test for screening of subclinical Cushing's syndrome in patients with adrenal incidentalomas. Journal of Endocrinological Investigation 201134 e1-e5. (https://doi.org/10.1007/BF03346701)

24 Baid SK, Rubino D, Sinaii N, Ramsey S, Frank A \& Nieman LK. Specificity of screening tests for Cushing's syndrome in an overweight and obese population. Journal of Clinical Endocrinology \& Metabolism 200994 3857-3864. (https://doi.org/10.1210/jc.2008-2766)

25 Hong AR, Kim JH, Hong ES, Kim IK, Park KS, Ahn CH, Kim SW, Shin CS \& Kim SY. Limited diagnostic utility of plasma adrenocorticotropic hormone for differentiation between adrenal Cushing syndrome and Cushing disease. Endocrinology \& Metabolism 201530 297-304. (https://doi.org/10.3803/EnM.2015.30.3.297) 
26 Terzolo M, Pia A \& Reimondo G. Subclinical Cushing's syndrome: definition and management. Clinical Endocrinology 201276 12-18. (https://doi.org/10.1111/j.1365-2265.2011.04253.x)

27 Schneller J, Reiser M, Beuschlein F, Osswald A, Pallauf A, Riester A, Tietze JK, Reincke M \& Degenhart C. Linear and volumetric evaluation of the adrenal gland-MDCT-based measurements of the adrenals. Academic Radiology 201421 1465-1474. (https://doi. org/10.1016/j.acra.2014.06.008)

28 Giraldi FP \& Ambrogio AG. Variability in laboratory parameters used for management of Cushing's syndrome. Endocrine $2015 \mathbf{5 0} 580-589$. (https://doi.org/10.1007/s12020-015-0676-9)

29 Giraldi FP, Saccani A \& Cavagnini F. Assessment of ACTH assay variability: a multicenter study. European Journal of Endocrinology 2011 164 505-512. (https://doi.org/10.1530/EJE-10-0962)

30 Carnes M, Goodman BM, Lent SJ, Vo H \& Jaeckels R. Coincident plasma ACTH and corticosterone time series: comparisons between young and old rats. Experimental Gerontology 199429 625-643. (https://doi.org/10.1016/0531-5565(94)90075-2)

31 Kim BY, Won JC, Lee JH, Kim HS, Park JH, Ha KH, Won KC, Kim DJ \& Park KS. Diabetes fact sheets in Korea, 2018: an appraisal of current status. Diabetes \& Metabolism Journal 201943 487-494. (https://doi. org/10.4093/dmj.2019.0067)

32 Reimondo G, Castellano E, Grosso M, Priotto R, Puglisi S, Pia A, Pellegrino M, Borretta G \& Terzolo M. Adrenal incidentalomas are tied to increased risk of diabetes: findings from a prospective study.
Journal of Clinical Endocrinology \& Metabolism 2020105 e973-e981. (https://doi.org/10.1210/clinem/dgz284)

33 Terzolo M, Pia A, Alì A, Osella G, Reimondo G, Bovio S, Daffara F, Procopio M, Paccotti P, Borretta G, et al. Adrenal incidentaloma: a new cause of the metabolic syndrome? Journal of Clinical Endocrinology \& Metabolism 200287 998-1003. (https://doi. org/10.1210/jcem.87.3.8277)

34 Reincke M, Fassnacht M, Väth S, Mora P \& Allolio B. Adrenal incidentalomas: a manifestation of the metabolic syndrome? Endocrine Research 199622 757-761. (https://doi. org/10.1080/07435809609043773)

35 Elhassan YS, Alahdab F, Prete A, Delivanis DA, Khanna A, Prokop L, Murad MH, O'Reilly MW, Arlt W \& Bancos I. Natural history of adrenal incidentalomas with and without mild autonomous cortisol excess: a systematic review and meta-analysis. Annals of Internal Medicine 2019171 107-116. (https://doi.org/10.7326/M183630)

36 Mazziotti G, Gazzaruso C \& Giustina A. Diabetes in Cushing syndrome: basic and clinical aspects. Trends in Endocrinology \& Metabolism 201122 499-506. (https://doi.org/10.1016/j. tem.2011.09.001)

37 Rubin RT \& Phillips JJ. Adrenal gland volume determination by computed tomography and magnetic resonance imaging in normal subjects. Investigative Radiology 199126 465-469. (https://doi. org/10.1097/00004424-199105000-00015)

Received 1 April 2020

Revised version received 4 June 2020

Accepted 29 June 2020 